\title{
Cardiac Biomarkers Predicting MACE in Patients Undergoing Noncardiac Surgery: A Meta-Analysis
}

\author{
Li-Jun Zhang ${ }^{1}$, Na Li ${ }^{1}$, Yang $\mathrm{Li}^{2,3}$, Xian-Tao Zeng ${ }^{2,3}$ and Mei-Yan Liu ${ }^{1 *}$ \\ ' Department of Cardiology, Beijing Anzhen Hospital, Capital Medical University, Beijing, China, ${ }^{2}$ Center for Evidence-Based \\ and Translational Medicine, Zhongnan Hospital of Wuhan University, Wuhan, China, ${ }^{3}$ Center for Evidence-Based and \\ Translational Medicine, Wuhan University, Wuhan, China
}

\section{OPEN ACCESS}

Edited by:

Ovidiu Constantin Baltatu, Anhembi Morumbi University, Brazil

Reviewed by:

Marion Hofmann Bowman, University of Michigan, United States Zoe Polizopoulou, Aristotle University of Thessaloniki, Greece

${ }^{*}$ Correspondence: Mei-Yan Liu liumeiyanaz@163.com

Specialty section:

This article was submitted to Integrative Physiology, a section of the journal Frontiers in Physiology

Received: 17 September 2018 Accepted: 20 December 2018 Published: 18 January 2019

Citation:

Zhang L-J, Li N, Li Y, Zeng X-T and Liu M-Y (2019) Cardiac Biomarkers Predicting MACE in Patients Undergoing Noncardiac Surgery: A Meta-Analysis. Front. Physiol. 9:1923.

doi: 10.3389/fphys.2018.01923
Objective: The present meta-analysis was aimed to systematically evaluate the effectiveness and accuracy of brain natriuretic peptide (BNP), cardiac troponin (cTn), high sensitive $\mathrm{C}$ reactive protein (hs-CRP) and CRP for predicting postoperative major adverse cardiovascular events (MACE) in patients undergoing noncardiac surgery.

Methods: A total of 26 relevant studies with 7,877 participants were collected from five databases, namely PubMed, Embase, China National Knowledge Infrastructure (CNKI), CQVIP and the Wanfang Database until August 10, 2018. And the Review Manager Version 5.3 and Stata/SE 12 software were used for data syntheses in the meta-analysis.

Results: Strong relationships of BNP/NT-proBNP, cTnl/cTnT and hs-CRP with MACE were detected in patients undergoing noncardiac surgery, and the five biomarkers all increased the risk of MACE. Compared to normal levels, elevated BNP/NT-proBNP could increase the MACE risk by almost 4-fold [RR:3.92, 95\%Cl: 3.23-4.75, $P<0.001$ ]; elevated BNP corresponded to a 4.5-fold risk [RR:4.57, 95\%Cl: 3.37-6.20, $P<0.001$ ]; elevated NT-proBNP led to a 3-fold higher risk [RR:3.48, 95\%Cl: 2.71-4.46, $P<0.001]$. Comparing with normal levels of cTnl/cTnT, increased CTnl/cTnT was associated with nearly 5-fold more higher risk of MACE [RR:5.52, 95\%Cl: 4.62-6.58, $P<0.001]$; elevated cTnl faced a 5-fold risk [RR:5.21, 95\%Cl: 3.96-6.86, $P<0.001]$; elevated CTnT resulted in nearly 6-fold higher risk [RR:5.73, 95\%Cl: 4.55-7.22, $P<0.001]$. The elevation of hs-CRP was associated with nearly 4-fold higher risk of MACE in comparison with normal concentration [RR:3.73, 95\% Cl: 2.63-5.30, $P<0.001]$

Conclusion: According to the results of our meta-analysis, the elevations of BNP/NT-proBNP, cTnl/cTnT, and hs-CRP, pre-operation or post-operation immediately, can predict much higher risk of postoperative MACE in patients undergoing noncardiac surgery.

Keywords: cardiac biomarkers, cardiac risk factors and prevention, acute coronary syndromes, noncardiac surgery, meta-analysis

\section{INTRODUCTION}

Although the rate of perioperative events shows a declining tendency in the past 30 years, a consequence of the developments in anesthesiology and surgical techniques, cardiovascular complications still represent a leading cause of perioperative mortality in individuals receiving large noncardiac surgery (Devereaux et al., 2005). At the moment, the main method to assess adverse 
cardiovascular events lies in the evaluation of various risk factors, and predicted high-risk patients need undergo more detailed examinations during perioperative period, such as echocardiography and cardiac angiography. Therefore, it is important to formulate a cost-effective, convenient and accurate method for preoperative evaluation, risk stratification and optimization prior surgery, and the use of cardiac biomarkers has been suggested for this role. Biomarkers may help to reduce the morbidity rate of major adverse cardiovascular event (MACE, including death, stroke, myocardial infarction, and need for coronary artery bypass graft or percutaneous coronary intervention) during perioperative and postoperative period.

Cardiac biomarkers, such as brain natriuretic peptide (BNP) and amino-terminal pro-hormone of brain natriuretic peptide (NT-proBNP), cardiac troponins ( $\mathrm{cTn}$ ), high sensitive $\mathrm{C}$ reactive protein (hs-CRP) and CRP, are currently considered to play a pivotal role in evaluating treatment strategy and prognosis of different cardiac diseases (McMurray et al., 2012; Clerico et al., 2014; Thygesen et al., 2018). European Society of Cardiology and European Society of Anesthesiology guidelines for preoperative cardiac risk assessment have recommended that preoperative BNP or NT-proBNP measurement should be considered in high-risk patients undergoing noncardiac surgery (Thygesen et al., 2018). In addition, BNP would increase during exercise in patients without heart failure and healthy individuals, resulting from transient myocardial wall stress, cardiomyocyte metabolic changes, and neuroendocrinology response (Hamasaki, 2016).

In recent years, a large number of studies have verified the value of preoperative BNP or NT-proBNP, cTnI/cTnT, and hs-CRP/CRP for cardiovascular risk prediction in patients undergoing noncardiac surgery (McMurray et al., 2012; Clerico et al., 2014). The aim of our study was to evaluate the correlation between preoperative cardiac biomarkers and perioperative MACE in noncardiac surgery patients through the method of meta-analysis, with the hope of providing better evidence for clinical application.

\section{METHODS}

\section{Search Strategy}

We methodically searched the databases of PubMed, Embase, China National Knowledge Infrastructure (CNKI), VIP China Science, and Wanfang with the combination of the following search keywords: "b-type natriuretic peptide" or "Natriuretic Peptide, Brain" or "high-sensitivity C reactive protein" or "hsCRP" or "C reactive protein" or "CRP" or "Troponin." "cardiac events" or "cardiovascular events" or "major adverse cardiac events," and "surgery." Publication languages were limited to English and Chinese only. Literature was restricted to those published before December 09, 2018. References of relevant articles were further screened to identify additional eligible studies. The principal authors of reports lacking essential data would be contacted to obtain detailed information for our metaanalysis.

\section{Data Extraction}

Relevant articles were selected independently by two experienced investigators based on the following criteria: (1) participants' age $\geq 18$ years, (2) with a cohort study design, (3) stating the level of cardiac biomarkers detected before or immediately after operation, (4) study subjects only receiving noncardiac surgery, (5) providing sufficient information for the calculation of risk ratio (RR), and (6) offering complete text.

Quality assessment: All of the included articles were reviewed in detail. Essential information was independently extracted by two experienced investigators, and discrepancies over these data between the two investigators were resolved through discussion to reach a final consensus. When two or more articles used the same group of original population data, only the article with the largest sample size was included in this meta-analysis. The Newcastle-Ottawa Scale (NOS) 24 was used to assess the quality of all included articles (Stang, 2010). This Scale has three parts: selection, comparability, and exposure, containing eight items. The total score ranges from zero to nine stars. We classified articles with seven to nine stars as high quality, those with five to six stars as medium quality, and those with zero to four stars as poor quality. And articles with poor quality were excluded.

\section{Detail Information About MACE}

Patients undergoing noncardiac surgeries are at high risk of MACE, which is a significant clinical and economical challenge. MACE mentioned in this meta-analysis included heart failure, acute coronary syndrome, atrial fibrillation, paroxysmal supraventricular tachycardia, ventricular tachycardia, angina pectoris, acute myocardial infarction, thromboembolic events, deep vein thrombosis, acute renal failure, transient ischemic attack, cardiac death, all-cause mortality, major arrhythmia, unstable angina, stroke, cardiac revascularization procedure.

\section{Statistical Analysis}

Data were analyzed using Review Manager (RevMan) Version 5.3 (The Nordic Cochrane Center, Copenhagen, Denmark) and Stata/SE 12 (StataCorp., College Station, Texas). Risk ratio (RR) with $95 \%$ confidence $(95 \% \mathrm{CI})$ was calculated to measure effect size. Heterogeneity was evaluated with Cochran's Q-test (DerSimonian and Laird, 1986) and $\mathrm{I}^{2}$ statistic (Melsen et al., 2014), and would be considered as insignificant to moderate when $\mathrm{I}^{2}=0-50 \%$ while as statistically significant when $\mathrm{I}^{2}>$ $50 \%$. Random-effects model would be used for calculating pooled effect size when heterogeneity was significant $(P<0.10$ or $\mathrm{I}^{2}>50 \%$ ), while fixed-effects model would be utilized in the absence of significant heterogeneity $\left(P>0.10\right.$ or $\left.\mathrm{I}^{2}<50 \%\right)$. Considering the discrepancies in measured cardiac biomarkers across the studies, stratified analyses were performed in terms of biomarkers' types and methods for biomarker measurement. In addition, sensitivity analyses were performed via excluding studies one at a time (Tobias, 1999). Funnel plots were adopted to detect publication bias (Begg and Mazumdar, 1994). Pvalues were two-sided, and $<0.05$ was considered statistically significant. 


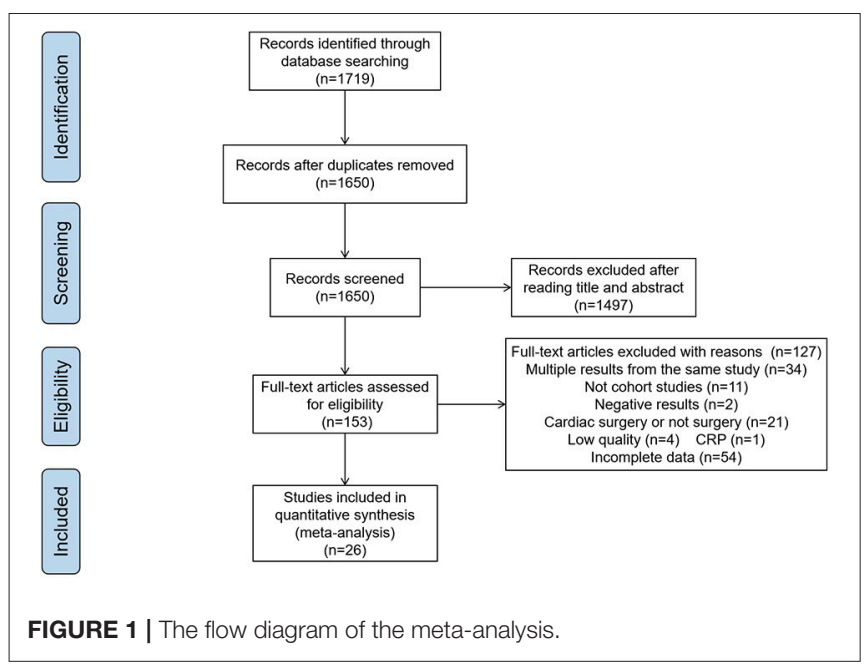

\section{RESULTS}

\section{Literature Selection}

Initially, 1,719 publications were obtained from the databases. After removing duplicates and reviews, 1,650 articles were left. In the examinations on titles and abstracts, 1,497 articles were excluded. Of the remaining 153 papers, 26 articles were considered to be eligible for our study after reading the full texts, and the other 127 articles were excluded for the following reasons: multiple results from the same study $(n=34)$, not cohort studies $(n=11)$, negative results $(n=2)$, cardiac surgery $(n=$ $21)$, low quality $(n=4)$, incomplete data $(n=54)$, only 1 article for CRP, so we excluded it $(n=1)$. (Figure 1)

Study Characteristics: A total of 7,877 participants were recruited in the eligible articles Among them three articles (Oscarsson et al., 2009b; Stone et al., 2014; Kopec et al., 2017) reported two biomarkers, eleven studies detected an association between BNP/ NT-proBNP and MACE (Feringa et al., 2007; Rajagopalan et al., 2008, 2011; Oscarsson et al., 2009a,b; Vetrugno et al., 2012; Cagini et al., 2014; Stone et al., 2014; Kim et al., 2016; Long et al., 2016; Kopec et al., 2017), fourteen reported an association between cTn and MACE (Lopez-Jimenez et al., 1997; Oscarsson et al., 2004, 2009b; Gibson et al., 2006; Ausset et al., 2008; Dawson-Bowling et al., 2008; Chong et al., 2009; Winkel et al., 2010; Biccard et al., 2012; Shroff et al., 2012; Gillmann et al., 2014; Kopec et al., 2017; Golubović et al., 2018; Kim et al., 2018), and four revealed a relationship between hs-CRP and MACE (Owens et al., 2007; Martins et al., 2011; Scrutinio et al., 2011; Stone et al., 2014; Table 1). The quality evaluation according to NOS of each selected article was also shown in Table 1. The studies included in this meta-analysis were all cohort studies, including 1 nested cohort study (Kopec et al., 2017), 5 retrospective studies (Shroff et al., 2012; Stone et al., 2014; Kim et al., 2016, 2018; Long et al., 2016) and others were prospective studies. Meanwhile, Kim reported NT-proBNP, Kopec reported NT-proBNP and cTnT, Long and Stone reported BNP, Shroff and Kim reported cTnI, and Stone also reported hs-CRP.

\section{BNP/NT-proBNP and MACE}

The fixed-effects model was utilized to pool summary effects for BNP/NT-proBNP $\left(P=0.06, \mathrm{I}^{2}=44 \%\right)$. Strong relationship was detected between BNP/NT-proBNP and MACE in patients undergoing noncardiac surgery, and BNP and NT-proBNP both increased MACE risk. Comparing with normal BNP/NTproBNP, elevated BNP/NT-proBNP was associated with an almost 4-fold higher risk of MACE [RR: 3.92, 95\%CI: 3.23-4.75, $P$ $<0.001]$. Subgroup analysis showed that elevated BNP increased MACE risk by 4.5-fold when compared to normal BNP [RR:4.57, 95\%CI: 3.37-6.20, $P<0.001$ ], and elevated NT-proBNP led to a more than 3-fold higher risk of MACE when compared to normal NT-proBNP [RR:3.48, 95\%CI: 2.71-4.46, $P<0.001$ ] (Figure 2). In the subgroup analysis of BNP regarding different study types, retrospective studies showed elevated BNP increased MACE risk by 5 -fold when compared to normal BNP [RR:5.24, 95\%CI:3.617.61, $P<0.001]$, prospective studies showed elevated BNP increased MACE risk by 3-fold when compared to normal BNP [RR:3.39, 95\%CI:1.98-5.79, $P<0.001]$.

\section{cTnl/cTnT and MACE}

The fixed-effects model was employed for data pooling $(P=$ $0.51, \mathrm{I}^{2}=0 \%$ ). According to summarized results, $\mathrm{cTnI}$, and $\mathrm{cTnT}$ could strongly increase the risk of MACE in patients undergoing noncardiac surgery. Comparing with normal $\mathrm{cTnI} / \mathrm{cTnT}$, the elevation of $\mathrm{cTnI} / \mathrm{cTnT}$ was associated with nearly 5 -fold more higher risk of MACE [RR:5.52, 95\%CI: 4.62-6.58, $P<0.001$ ]. In stratified analysis, elevated cTnI conferred a 5-fold risk [RR:5.21, 95\%CI: 3.96-6.86, $P<0.001]$; when compared to normal cTnI, and elevated cTnT resulted in nearly 6 -fold higher risk when compared to normal cTnT [RR:5.73, 95\%CI: 4.55-7.22, $P<$ 0.001] (Figure 3). In the subgroup analysis of cTnI regarding different study types, retrospective studies showed elevated cTnI increased MACE risk by 5 -fold when compared to normal cTnI [RR:5.49, 95\%CI:3.76-8.03, $P<0.001$ ], prospective studies showed elevated cTnI increased MACE risk by 9 -fold when compared to normal cTnI [RR:5.04, 95\%CI:3.45-7.37, $P<0.001]$.

\section{hs-CRP and MACE}

The fixed-effects model was utilized for pooling data $(P=0.59$, $\left.\mathrm{I}^{2}=0.0 \%\right)$. The results showed a strong relationship between hs-CRP and increased MACE in patients undergoing noncardiac surgery. Comparing with normal hs-CRP, the elevation of hsCRP could increase MACE risk by nearly 4-fold [RR: 3.73, 95\%CI: 2.63-5.30, $P<0.001$ ] (Figure 4).

\section{Sensitivity Analysis}

Sensitivity analysis was performed through excluding a single study each time to detect the influence of individual datasets on pooled RRs. The analysis results for BNP and NT-proBNP suggested that the power of pooled RRs was decreased after excluding Long G's study (Long et al., 2016) while increased after excluding Feringa HH's (Feringa et al., 2007) or Oscarsson A's study (Oscarsson et al., 2009a). We considered the potential reasons for the analysis results included: (1) Long G's study was a retrospective and enrolled the most patients than other studies; (2) Oscarsson A's study had the fewest participants than other 


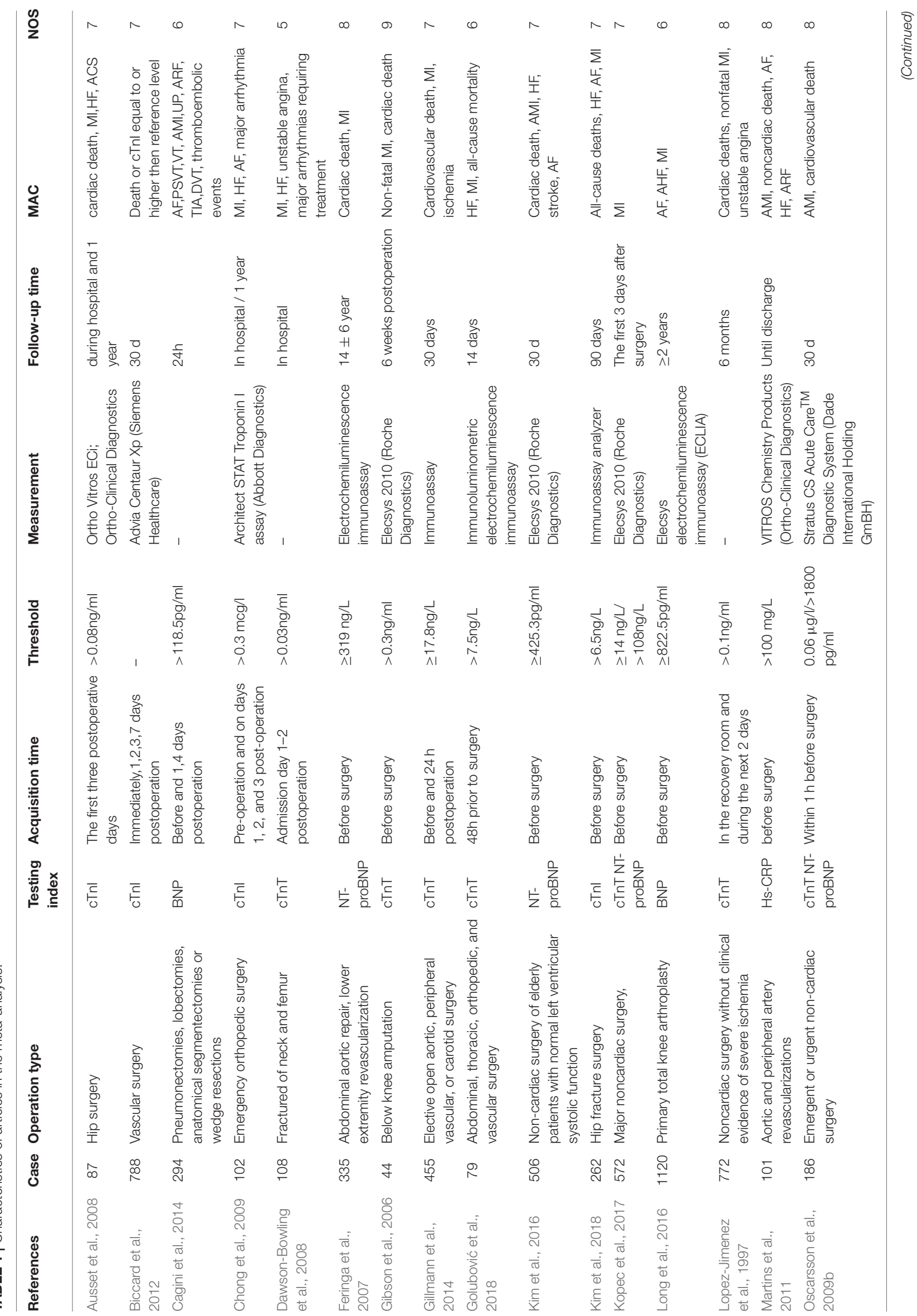




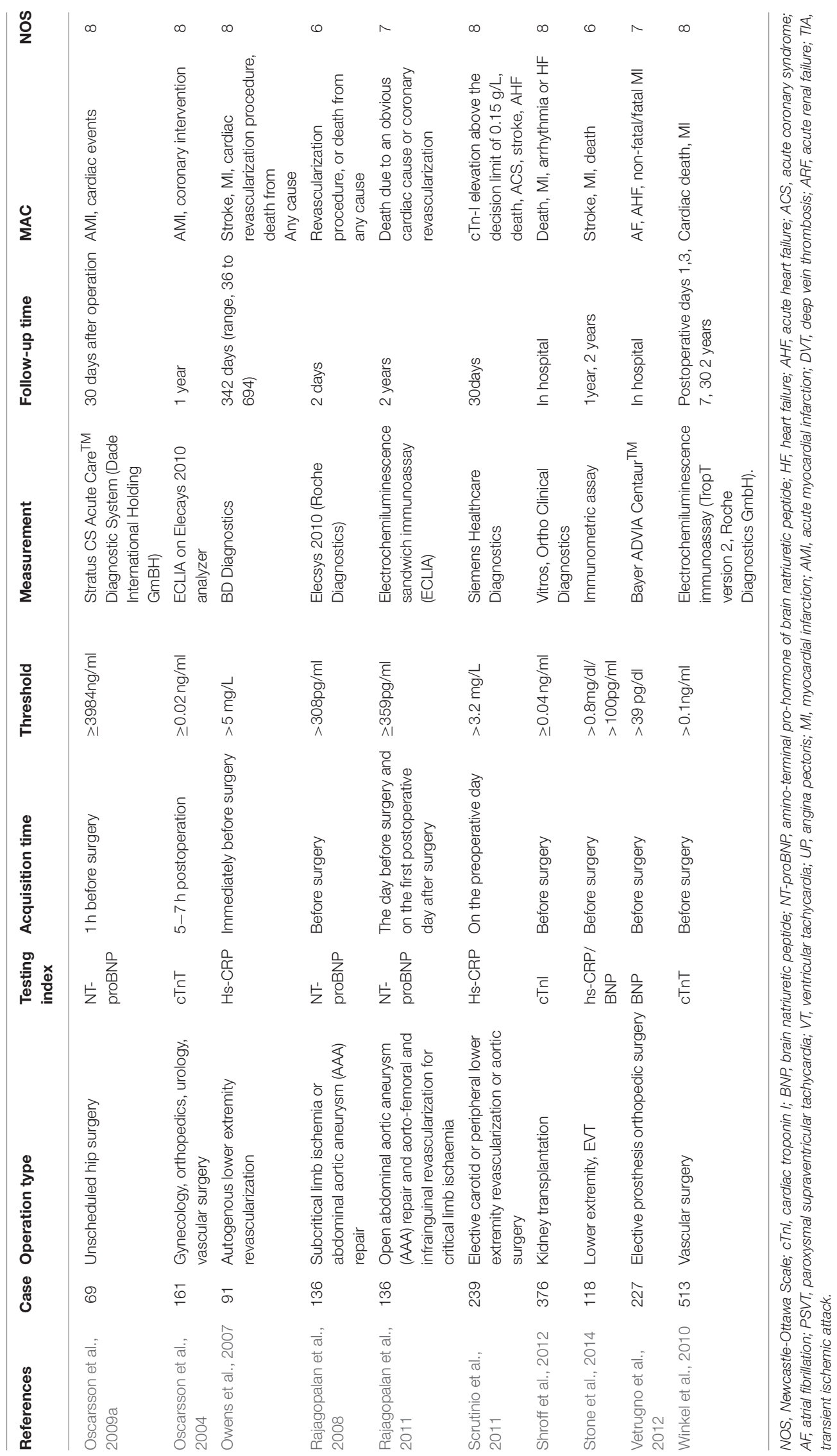




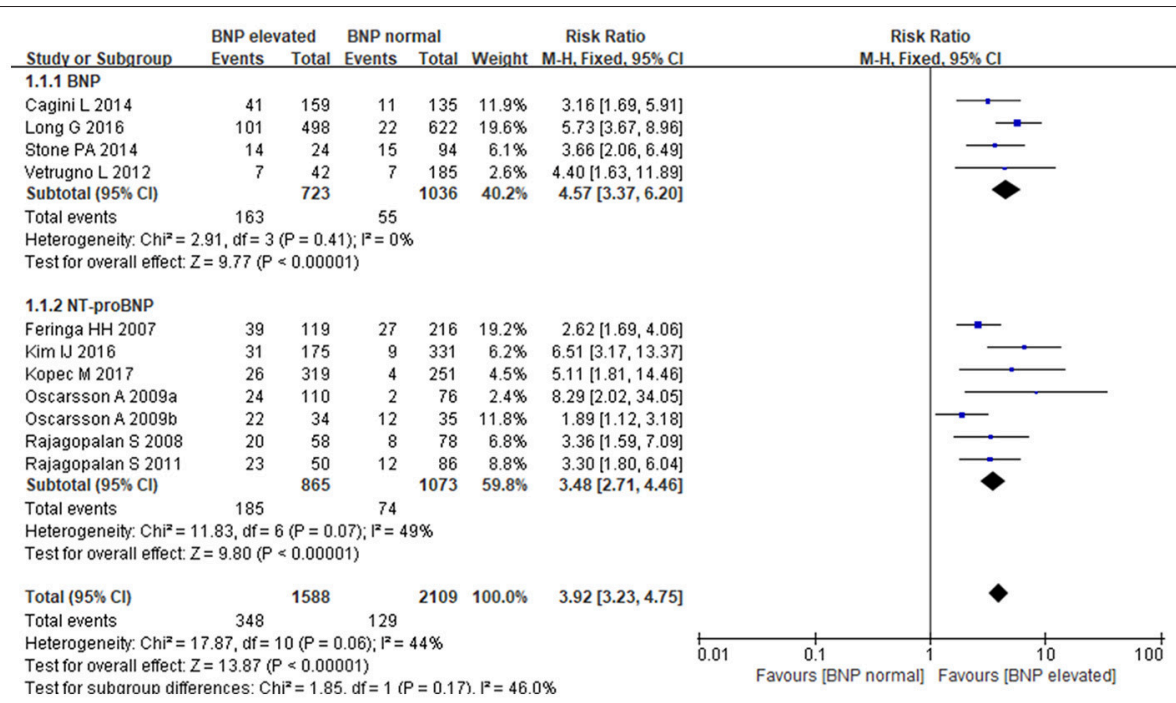

FIGURE 2 | The association between BNP/NT-proBNP and MACE.

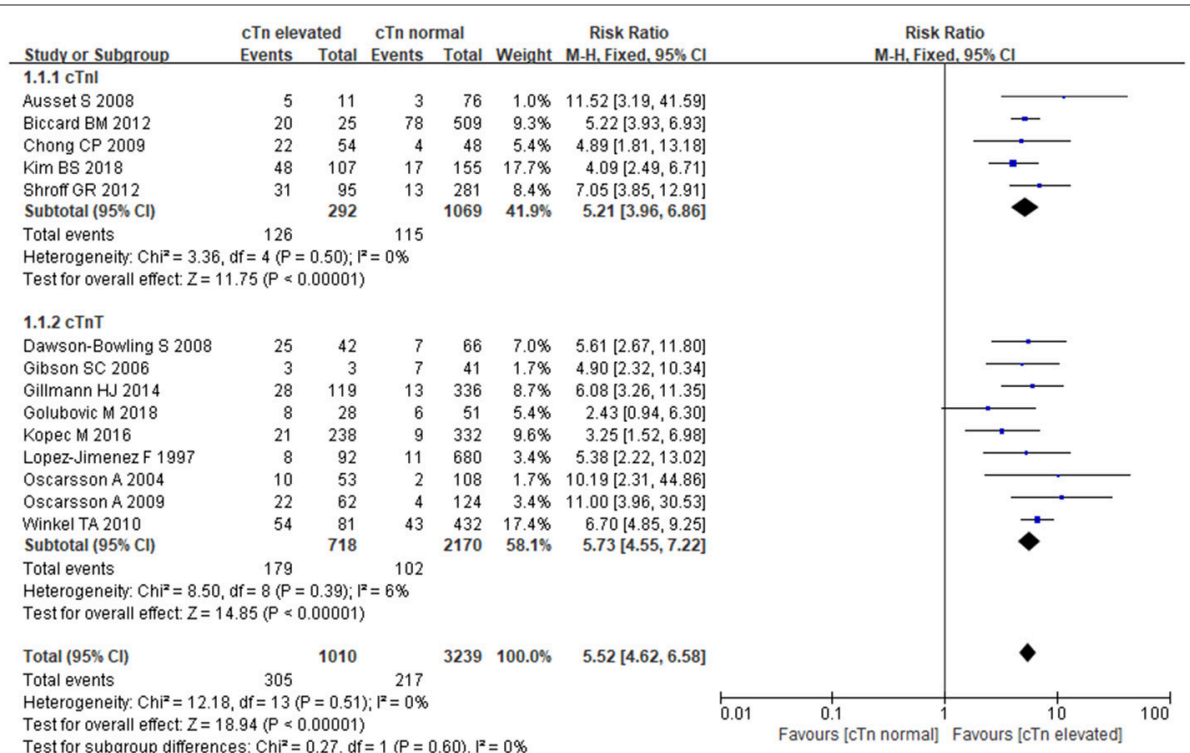

FIGURE 3 | The association between cTnl/cTnT and MACE.

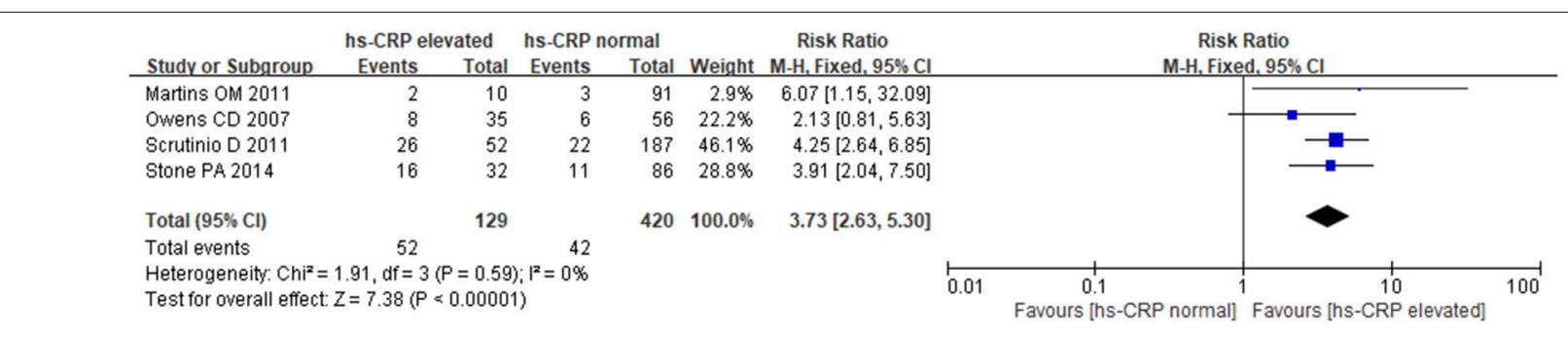

FIGURE 4 | The association between hs-CRP and MACE. 

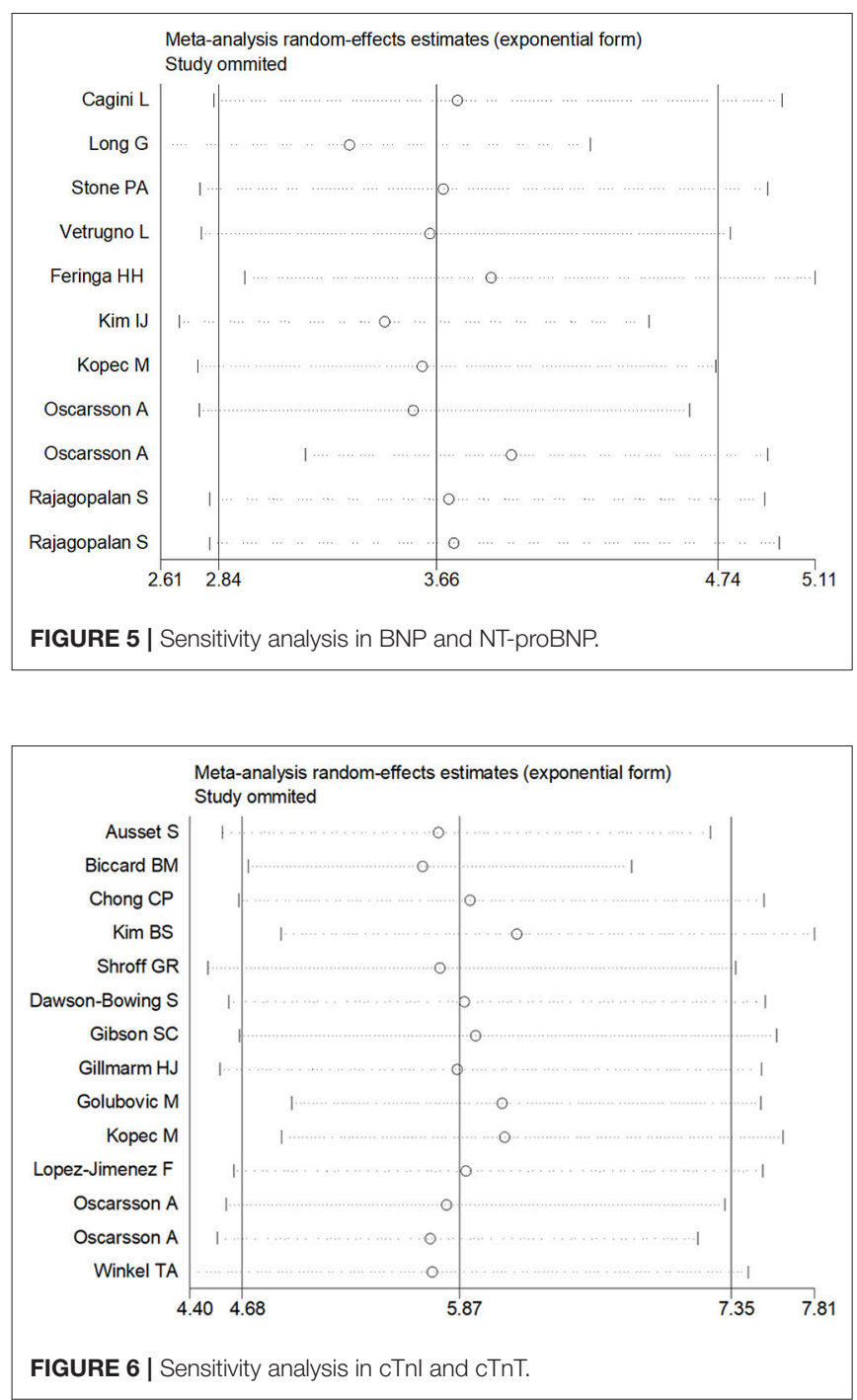

studies; (3) Compared with other studies, there was minimum difference of MACE incidence between BNP elevated group and BNP normal group in Feringa HH's study. Nonetheless, the RRs were not altered when removing any one of the other studies. The analysis for $\mathrm{cTnI}, \mathrm{cTnT}$ and hs-CRP found no obvious alterations the final pooled RRs during the exclusion of any studies. The results from sensitivity analysis indicated that our results were robust (Figures 5, 6).

\section{Publication Bias}

The funnel plot for BNP/NT-proBNP, cTnI/cTnT, and hsCRP showed no asymmetry, indicating publication bias was insignificant in this meta-analysis. Besides, such inference was further supported by statistical evidence from modified Begg's tests. Therefore, there was no significant publication bias between included studies in this meta-analysis $(P=0.39$ for BNP/NTproBNP; $P=0.23$ for $c$ TnI/cTnT; $P=0.96$ for hs-CRP) (Figure 7).

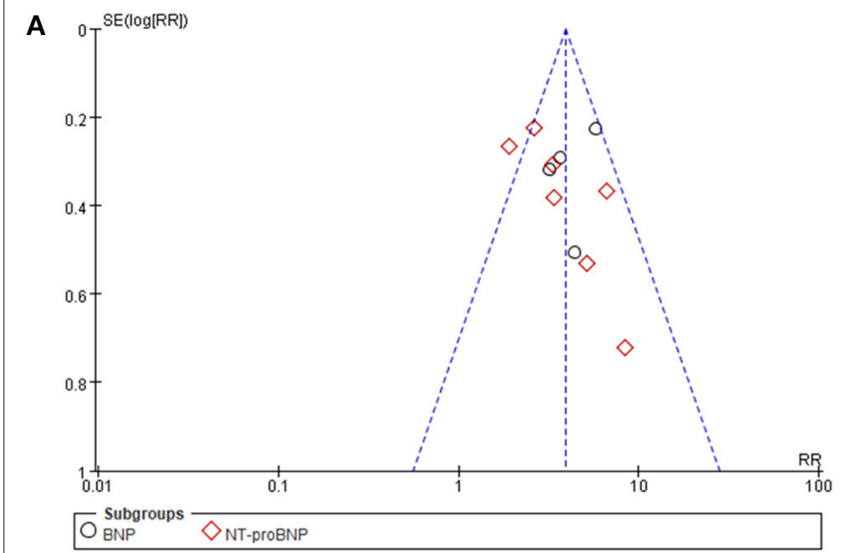

B

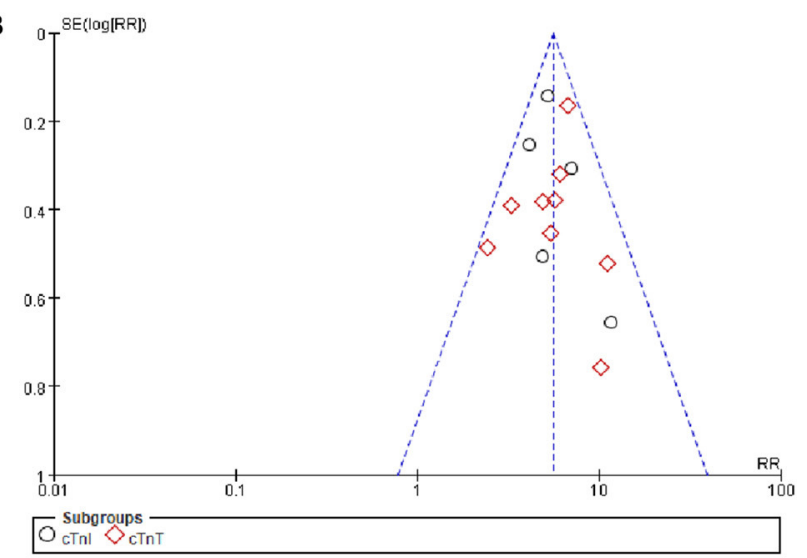

c

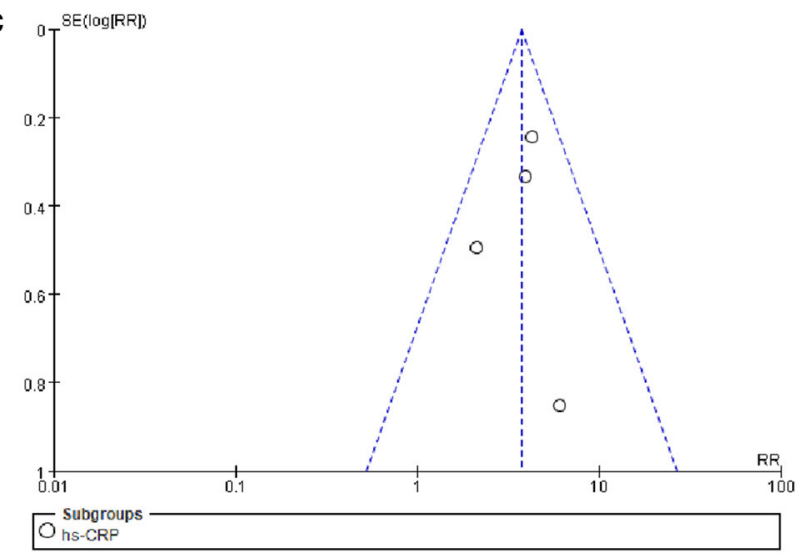

FIGURE 7 | (A-C) Begg's funnel plot with pseudo 95\% confidence limits.

\section{DISCUSSION}

In this meta-analysis, we found a strong relationship of BNP/NTproBNP [RR:3.92, 95\%CI: 3.23-4.75, $P<0.001$ ] (Feringa et al., 2007; Rajagopalan et al., 2008, 2011; Oscarsson et al., 2009a,b; Vetrugno et al., 2012; Cagini et al., 2014; Stone et al., 2014; Kim et al., 2016; Long et al., 2016; Kopec et al., 2017), cTnI/cTnT [RR:5.85, 95\%CI: 4.85-7.06, $P<0.001$ ] (Lopez-Jimenez et al., 1997; Oscarsson et al., 2004, 2009b; Gibson et al., 2006; Ausset et al., 2008; Dawson-Bowling et al., 2008; Chong et al., 2009; 
Winkel et al., 2010; Biccard et al., 2012; Shroff et al., 2012; Gillmann et al., 2014; Kopec et al., 2017; Golubović et al., 2018; Kim et al., 2018), and hs-CRP [RR:3.73, 95\%CI: 2.63-5.30, $P<$ 0.001] (Owens et al., 2007; Martins et al., 2011; Scrutinio et al., 2011; Stone et al., 2014) with MACE in patients undergoing noncardiac surgery, and all of these biomarkers were positively related to the risk of MACE.

It has been reported that patients undergone noncardiac surgeries are at high risk of MACE due to multiple comorbidity (Kumar et al., 2001). Elderly patients after hip fracture surgery have higher mortality (Oscarsson et al., 2009a). Post-operative MI often occurs silently and come up with poor prognosis (Roberts and Goldacre, 2003). Almost one third of patients suffered myocardial ischaemia in the perioperative period of fractured neck of femur surgery (Roberts and Goldacre, 2003). Therefore, biomarkers detection could be a potential cardiac assessment for cardiac events.

Though different study types may cause certain bias, we found no significant difference caused by study types in this metaanalysis. All the studies included in this meta-analysis were cohort studies, including 1 nested cohort study (Kopec et al., 2017), 5 retrospective studies (Shroff et al., 2012; Stone et al., 2014; Kim et al., 2016, 2018; Long et al., 2016) and others were prospective studies. Meanwhile, Kim reported NT-proBNP, Kopec reported NT-proBNP and cTnT, Long and Stone reported BNP, Shroff and Kim reported cTnI, and Stone also reported hsCRP. We made a subgroup analysis according to study type in $\mathrm{BNP}$ and $\mathrm{cTnI}$. The results showed no difference in retrospective studies and prospective studies. In the sensitivity analyses of NT-proBNP, cTnT, and hs-CRP, we could see no significant difference was found after removing Kim, Kopec, and Stone's studies respectively.

Both NT-proBNP and BNP are derived from the cleavage of BNP precursor, but NT-proBNP has longer half-life, higher plasma concentration and more stable biological properties than BNP, though both are associated with a variety of heart diseases such as left ventricular dysfunction, valvular heart disease, and acute coronary syndrome (Weber and Hamm, 2006). BNPs are secreted from the myocardium in response to multiple stimuli such as ischaemia, myocardial stretch, inflammation and other neuroendocrine stimuli (Clerico et al., 2011). The level of BNP or NT-proBNP may provide guidance for preoperative preparation, especially for the assessment of ventricular systolic and diastolic function, and they even may reflect minor changes in ventricular function induced by transient myocardial ischemia. Recent studies have found lower BNP and NT-ProBNP levels in patients with heart failure with preserved ejection fraction (HFPEF) than those with reduced ejection fraction (HFREF) (Bishu et al., 2012). Moreover, Ishigaki T et al (Ishigaki et al., 2015) demonstrated the different implications of elevated BNP in HFPEF and HFREF, the former may be associated with high filling pressure, while the latter may be related with myocardial dysfunction. Meanwhile, plasma BNP levels can also reflect the risk and severity of coronary artery lesions in patients with stable coronary heart disease (Niizuma et al., 2009). Therefore, it is reasonable to choose BNP or NT- proBNP level as predictor for postnoncardiac surgery cardiovascular events. Lucio Cagini et al.
(2014) demonstrated that increased BNP first days after thoracic surgery including pneumonectomies, lobectomies, anatomical segmentectomies or wedge resections, resulted in 3-fold higher risk of developing postoperative cardiopulmonary complications. In patients with hip fractures, Oscarsson et al. (2009a) found that the elevation of preoperative NT-proBNP was strongly connected with cardiac complications in perioperative period. Stone et al. (2017) had also proven the significant role of BNP in predicting MACE in patients undergoing carotid intervention, however, due to the incomplete data, we haven't included this paper. In consistent with the conclusions of previous studies, our metaanalysis unveils that both BNP and NT-proBNP are valuable predictors for MACE in postoperative period.

Troponin (Tn) is a structural protein of cardiac contractility and a part of the muscle calcium tropomyosin complex, showing a major role in skeletal and cardiac muscle contraction. There are three subunits in troponin complex: TnI, TnT, and TnC. Our study mainly focused on cTnI and cTnT for they are highly sensitive markers of myocardial injury. Three to six hour after the damage to cardiac muscle cells, troponins can be detected in patient's blood and remain raised for 4-10 days. It has been widely recognized that elevated $c \mathrm{TnT}$ and $\mathrm{cTnI}$ can help identify risks of future major cardiac events (Oscarsson et al., 2004). As a specific marker for myocardial cell injury, cTnI has high myocardial specificity, and has been widely used in ischemic myocardial injury. In recent years, both domestic and foreign scholars have devoted themselves to explorations on cTnI, which has been designated as the gold standard for MI diagnosis abroad (Haggart et al., 2001; Landesberg et al., 2003). Ausset et al demonstrated that troponin I elevation in the first three days after operation was associated with 10-fold higher risk of MACE based on 88 consecutive patients undergoing hip surgery (Ausset et al., 2008). After 6 months of follow-up for 772 patients receiving major noncardiac surgery, Lopez-Jimenez et al. (1997) concluded that patients with elevated cTnT had higher risk of facing cardiac events than those with normal cTnT. Our study also obtained high value for cTnI and cTnT in predicting MACE. In addition, Mochmann et al. (2016) found there was no association between cTn elevation with coronary culprit lesion in acute ischemic stroke patients. However, the pathophysiology of cTn elevation still needs further investigation (Hofmann Bowman and Liao, 2016).

Some large prospective studies have drawn consistent conclusion that CRP could be regarded as a cardiovascular risk factor as traditional risk factors (Kaptoge et al., 2010; Piepoli et al., 2017). In a prospective study enrolled 459 consecutive patients who received kidney transplantation, CRP showed a positive role in predicting cardiac events. (Kruger et al., 2010) However, to our disappointment, we couldn't include CRP in this metaanalysis for only this one suitable study was found. Hs-CRP is an enhanced sensitivity C-reactive protein (CRP) immunoassay with a lowered measurement cutoff, hence, hs-CRP has higher sensitivity than CRP, being measured by laser nephelometry. Moreover, hs-CRP is widely used in clinical practice as a vital inflammatory biomarker. Inflammation is considered to take place in the pathophysiological process of cardiovascular diseases, therefore, hs-CRP, as a vital inflammatory biomarker, 
would be relevant with cardiac events (Ridker et al., 1997; Owens et al., 2007). In a 15-month follow-up study, increased hsCRP plasma concentration was reported to be associated with unfavorable prognosis in non-ST elevation acute myocardial infarction (NSTEMI) patients with AF (Pavlovic et al., 2017). Nortamo et al. (2017) reported hs-CRP could predict the risk of new-onset $\mathrm{AF}$ in patients with $\mathrm{CAD}$. In a meta-analysis including 83,995 participants, Li et al. (2017) demonstrated that hs-CRP could be significant risk factor in predicting all-cause, cardiovascular mortality. Based on a total of 101 patients undergoing noncardiac surgery, Martins et al. (2011) demonstrated that elevated hs-CRP levels meant increased risk of perioperative acute myocardial infarction. In our meta-analysis, relevant results on the basis of four studies showed that hsCRP might be regarded as a predictor for MACE in noncardiac surgery patients, and that the higher hs-CRP levels, preoperation or postoperation immediately, the higher risk of developing postoperative MACE.

\section{Strength and Limitations}

To our knowledge, this is the first study to evaluate the relationship between five biomarkers (BNP, NT-proBNP, cTnI, cTnT, and hs-CRP) and MACE. After examinations, we found that all of these biomarkers could be used as the predictors for postoperative MACE, and such findings may have certain clinic value in protecting patients undergoing noncardiac surgery from perioperative MACE. In this meta-analysis, eligible studies involved numerous noncardiac surgeries, such as hip surgery, vascular surgery and kidney transplantation. However, there are some limitations: Firstly, it is impossible for us to incorporate all kinds of noncardiac surgeries in this meta-analysis. Secondly, it would be more persuasive to embrace more studies on the

\section{REFERENCES}

Ausset, S., Auroy, Y., Lambert, E., Vest, P., Plotton, C., Rigal, S., et al. (2008). Cardiac troponin I release after hip surgery correlates with poor long-term cardiac outcome. Eur. J. Anaesthesiol. 25, 158-164. doi: $10.1017 /$ S0265021507001202

Begg, C. B., and Mazumdar, M. (1994). Operating characteristics of a rank correlation test for publication bias. Biometrics 50, 1088-1101.

Biccard, B. M., Naidoo, P., and de Vasconcellos, K. (2012). What is the best pre-operative risk stratification tool for major adverse cardiac events following elective vascular surgery? a prospective observational cohort study evaluating pre-operative myocardial ischaemia monitoring and biomarker analysis. Anaesthesia 67, 389-395. doi: 10.1111/j.1365-2044.2011.07020.x

Bishu, K., Deswal, A., Chen, H. H., LeWinter, M. M., Lewis, G. D., Semigran, M. J., et al. (2012). Biomarkers in acutely decompensated heart failure with preserved or reduced ejection fraction. Am. Heart J. 164, 763-770 e763. doi: 10.1016/j.ahj. 2012.08.014

Cagini, L., Andolfi, M., Leli, C., Potenza, R., Ragusa, M., Scarnecchia, E., et al. (2014). B-type natriuretic peptide following thoracic surgery: a predictor of postoperative cardiopulmonary complications. Eur. J. Cardiothorac. Surg. 46, e74-80. doi: 10.1093/ejcts/ezu348

Chong, C. P., Lam, Q. T., Ryan, J. E., Sinnappu, R. N., and Lim, W. K. (2009). Incidence of post-operative troponin I rises and 1-year mortality after emergency orthopaedic surgery in older patients. Age Ageing 38, 168-174. doi: 10.1093/ageing/afn231 association between biomarker and MACE, but so far, relevant clinical studies were limited. Only 4 studies about hs-CRP were included in this meta-analysis, and we couldn't do a metaanalysis about CRP for only 1 study about CRP was found. We will follow the clinical study progression in this area and rewrite the meta-analysis in the future. In addition, consensus has not been achieved on how to define normal range and on how to use these indicators to classify preoperative risks. Studies on how to utilize perioperative cardiac biomarkers to predict MACE after non cardiac surgery need to be further improved, thus reducing the incidence of MACE after non cardiac surgery.

\section{CONCLUSION}

In conclusion, BNP, NT-proBNP, cTnI, cTnT and hs-CRP are all effective predictors for cardiovascular risk during noncardiac surgery. The elevation of these five biomarkers all increased the risk of MACE. Therefore, these biomarkers would be helpful for predicting MACE among patients undergoing noncardiac surgery.

\section{AUTHOR CONTRIBUTIONS}

M-YL contributed to the design of the paper. L-JZ contributed to data collection and paper revision. NL and YL contributed to data collection and analyses, and paper writing. X-TZ contributed to statistical analyses and paper revision.

\section{FUNDING}

This work was supported by the National Key Basic Research Program of China (973 program) Grant No. 2015 CB554402.

Clerico, A., Emdin, M., and Passino, C. (2014). Cardiac biomarkers and risk assessment in patients undergoing major non-cardiac surgery: time to revise the guidelines? Clin. Chem. Lab. Med. 52, 959-963. doi: 10.1515/cclm-20 13-0900

Clerico, A., Giannoni, A., Vittorini, S., and Passino, C. (2011). Thirty years of the heart as an endocrine organ: physiological role and clinical utility of cardiac natriuretic hormones. Am. J. Physiol. Heart Circ. Physiol. 301, H12-20. doi: 10. ajpheart.00226.201110.1152/ajpheart.00226.2011

Dawson-Bowling, S., Chettiar, K., Cottam, H., Worth, R., Forder, J., FitzgeraldO'Connor, I., et al. (2008). Troponin $\mathrm{T}$ as a predictive marker of morbidity in patients with fractured neck of femur. Injury 39, 775-780. doi: 10.1016/j.injury.2008.01.025

DerSimonian, R., and Laird, N. (1986). Meta-analysis in clinical trials. Control Clin. Trials 7, 177-188.

Devereaux, P. J., Goldman, L., Cook, D. J., Gilbert, K., Leslie, K., and Guyatt, G. H. (2005). Perioperative cardiac events in patients undergoing noncardiac surgery: a review of the magnitude of the problem, the pathophysiology of the events and methods to estimate and communicate risk. CMAJ 173, 627-634. doi: 10. 173/6/62710.1503/cmaj.050011

Feringa, H. H., Schouten, O., Dunkelgrun, M., Bax, J. J., Boersma, E., Elhendy, A., et al. (2007). Plasma N-terminal pro-B-type natriuretic peptide as longterm prognostic marker after major vascular surgery. Heart 93, 226-231. doi: 10.1136/hrt.2006.093716

Gibson, S. C., Marsh, A., Berry, C., Payne, C., Byrne, D. S., Rogers, P. N., et al. (2006). Should pre-operative troponin be a standard requirement in patients 
undergoing major lower extremity amputation? Eur. J. Vasc. Endovasc. Surg. 31, 637-641. doi: 10.1016/j.ejvs.2005.11.020

Gillmann, H. J., Meinders, A., Grohennig, A., Larmann, J., Bunte, C., Calmer, S., et al. (2014). Perioperative levels and changes of high-sensitivity troponin $\mathrm{T}$ are associated with cardiovascular events in vascular surgery patients. Crit. Care Med. 42, 1498-1506. doi: 10.1097/CCM.00000000000 00249

Golubović, M., Jankovic, R., Sokolovic, D., Cosic, V., Maravic-Stojkovic, V., Kostic, T., et al. (2018). Preoperative midregional pro-adrenomedullin and highsensitivity troponin t predict perioperative cardiovascular events in noncardiac surgery. Med. Princ. Pract. 27, 278-284. doi: 10.1159/000488197

Haggart, P. C., Adam, D. J., Ludman, P. F., and Bradbury, A. W. (2001). Comparison of cardiac troponin I and creatine kinase ratios in the detection of myocardial injury after aortic surgery. Br. J. Surg. 88, 1196-1200. doi: 10.1046/j.0007-1323.2001.01854.x

Hamasaki, H. (2016). The effects of exercise on natriuretic peptides in individuals without heart failure. Sports 4:32. doi: 10.3390/sports4020032

Hofmann Bowman, M. A., and Liao, J. K. (2016). Relative lack of culprit and obstructive coronary lesions in patients with acute ischemic stroke and elevated cardiac troponin. Circulation 133, 1228-1229. doi: 10.1161/circulationaha.116.021795

Ishigaki, T., Yoshida, T., Izumi, H., Fujisawa, Y., Shimizu, S., Masuda, K., et al. (2015). Different implication of elevated B-type natriuretic peptide level in patients with heart failure with preserved ejection fraction and in those with reduced ejection fraction. Echocardiography 32, 623-629. doi: 10.1111/echo.12707

Kaptoge, S., Di Angelantonio, E., Lowe, G., Pepys, M. B., Thompson, S. G., Collins, R., et al. (2010). C-reactive protein concentration and risk of coronary heart disease, stroke, and mortality: an individual participant meta-analysis. Lancet 375, 132-140. doi: 10.1016/S0140-6736(09)61717-7

Kim, B. S., Kim, T. H., Oh, J. H., Kwon, C. H., Kim, S. H., Kim, H. J., et al. (2018). Association between preoperative high sensitive troponin I levels and cardiovascular events after hip fracture surgery in the elderly. J. Geriatr. Cardiol. 15, 215-221. doi: 10.11909/j.issn.1671-5411.2018.03.002

Kim, I. J., Moon, J. Y., Ko, E. J., Lim, Y. M., Kim, S. H., Yang, W. I., et al. (2016). Prognostic value of preoperative $\mathrm{N}$-terminal pro-brain natriuretic peptide in non-cardiac surgery of elderly patients with normal left ventricular systolic function. Geriatr. Gerontol. Int. 16, 1109-1116. doi: 10.1111/ggi.12597

Kopec, M., Duma, A., Helwani, M. A., Brown, J., Brown, F., Gage, B. F., et al. (2017). Improving prediction of postoperative myocardial infarction with highsensitivity cardiac troponin t and NT-proBNP. Anesth. Analg. 124, 398-405. doi: 10.1213/ANE.0000000000001736

Kruger, B., Walberer, A., Debler, J., Boger, C. A., Farkas, S., Reinhold, S. W., et al. (2010). Is inflammation prior to renal transplantation predictive for cardiovascular and renal outcomes? Atherosclerosis 210, 637-642. doi: 10.1016/j.atherosclerosis.2010.01.004

Kumar, R., McKinney, W. P., Raj, G., Heudebert, G. R., Heller, H. J., Koetting, M., et al. (2001). Adverse cardiac events after surgery: assessing risk in a veteran population. J. Gen. Intern. Med. 16, 507-518. doi: 10.1046/j.1525-1497.2001.016008507.x

Landesberg, G., Shatz, V., Akopnik, I., Wolf, Y. G., Mayer, M., Berlatzky, Y., et al. (2003). Association of cardiac troponin, CK-MB, and postoperative myocardial ischemia with long-term survival after major vascular surgery. J. Am. Coll. Cardiol. 42, 1547-1554. doi: 10.1016/j.jacc.2003.05.001

Li, Y., Zhong, X., Cheng, G., Zhao, C., Zhang, L., Hong, Y., et al. (2017). Hs$\mathrm{CRP}$ and all-cause, cardiovascular, and cancer mortality risk: a meta-analysis. Atherosclerosis 259, 75-82. doi: 10.1016/j.atherosclerosis.2017.02.003

Long, G., Hao, C., Li, G., Yang, Y., Hongzhong, Z., and Zhenhu, W. (2016). Predictive value of B-type natriuretic peptide (BNP) for adverse cardiac events in patients undergoing primary total knee arthroplasty (TKA). J. Orthop. Sci. 21, 826-830. doi: $10.1016 /$ j.jos.2016.08.003

Lopez-Jimenez, F., Goldman, L., Sacks, D. B., Thomas, E. J., Johnson, P. A., Cook, E. F., et al. (1997). Prognostic value of cardiac troponin T after noncardiac surgery: 6-month follow-up data. J. Am. Coll. Cardiol. 29, 1241-1245.

Martins, O. M., Fonseca, V. F., Borges, I., Martins, V., Portal, V. L., and Pellanda, L. C. (2011). C-Reactive protein predicts acute myocardial infarction during high-risk noncardiac and vascular surgery. Clinics 66, 773-776. doi: $10.1590 /$ S1807-59322011000500011
McMurray, J. J., Adamopoulos, S., Anker, S. D., Auricchio, A., Bohm, M., Dickstein, K., et al. (2012). ESC guidelines for the diagnosis and treatment of acute and chronic heart failure 2012: the task force for the diagnosis and treatment of acute and chronic heart failure 2012 of the european society of cardiology. Developed in collaboration with the Heart Failure Association (HFA) of the ESC. Eur. J. Heart Fail 14, 803-869. doi: 10.1093/eurheartj/ehs104 Melsen, W. G., Bootsma, M. C., Rovers, M. M., and Bonten, M. J. (2014). The effects of clinical and statistical heterogeneity on the predictive values of results from meta-analyses. Clin. Microbiol. Infect. 20, 123-129. doi: 10.1111/1469-0691.12494S1198-743X(14)60045-8.

Mochmann, H. C., Scheitz, J. F., Petzold, G. C., Haeusler, K. G., Audebert, H. J., Laufs, U., et al. (2016). Coronary Angiographic findings in acute ischemic stroke patients with elevated cardiac troponin: the troponin elevation in acute ischemic stroke (TRELAS) Study. Circulation 133, 1264-1271. doi: 10.1161/CIRCULATIONAHA.115.018547

Niizuma, S., Iwanaga, Y., Yahata, T., Goto, Y., Kita, T., Miyazaki, S., et al. (2009). Plasma B-type natriuretic peptide levels reflect the presence and severity of stable coronary artery disease in chronic haemodialysis patients. Nephrol. Dial. Transl. 24, 597-603. doi: 10.1093/ndt/gfn491

Nortamo, S., Ukkola, O., Lepojarvi, S., Kentta, T., Kiviniemi, A., Junttila, J., et al. (2017). Association of sST2 and hs-CRP levels with new-onset atrial fibrillation in coronary artery disease. Int. J. Cardiol. 248, 173-178. doi: 10.1016/j.ijcard.2017.07.022

Oscarsson, A., Eintrei, C., Anskar, S., Engdahl, O., Fagerstrom, L., Blomqvist, P., et al. (2004). Troponin T-values provide long-term prognosis in elderly patients undergoing non-cardiac surgery. Acta Anaesthesiol. Scand. 48, 1071-1079. doi: 10.1111/j.1399-6576.2004.00463.x

Oscarsson, A., Fredrikson, M., Sorliden, M., Anskar, S., and Eintrei, C. (2009a). Nterminal fragment of pro-B-type natriuretic peptide is a predictor of cardiac events in high-risk patients undergoing acute hip fracture surgery. $\mathrm{Br} . \mathrm{J}$. Anaesth. 103, 206-212. doi: 10.1093/bja/aep139.

Oscarsson, A., Fredrikson, M., Sorliden, M., Anskar, S., Gupta, A., Swahn, E., et al. (2009b). Predictors of cardiac events in high-risk patients undergoing emergency surgery. Acta Anaesthesiol. Scand. 53, 986-994. doi: 10.1111/j.1399-6576.2009.01971.x

Owens, C. D., Ridker, P. M., Belkin, M., Hamdan, A. D., Pomposelli, F., Logerfo, F. et al. (2007). Elevated C-reactive protein levels are associated with postoperative events in patients undergoing lower extremity vein bypass surgery. J. Vasc. Surg. 45, 2-9; discussion 9. doi: 10.1016/j.jvs.2006.08.048

Pavlovic, M., Apostolovic, S., Stokanovic, D., Momcilovic, S., JevtovicStoimenov, T., Zdravkovic, S. C., et al. (2017). The association between galectin-3 and hs-CRP and the clinical outcome after non-ST-elevation myocardial infarction with preexisting atrial fibrillation. Sci. Rep. 7:15106. doi: 10.1038/s41598-017-15265-010.1038/s41598-017-15265-0

Piepoli, M. F., Hoes, A. W., Agewall, S., Albus, C., Brotons, C., Catapano, A. L., et al. (2017). 2016 European guidelines on cardiovascular disease prevention in clinical practice. The Sixth Joint Task Force of the European Society of Cardiology and Other Societies on Cardiovascular Disease Prevention in Clinical Practice constituted by representatives of 10 societies and by invited experts. Developed with the special contribution of the European Association for Cardiovascular Prevention and Rehabilitation. G Ital. Cardiol. 18, 547-612. doi: $10.1714 / 2729.27821$

Rajagopalan, S., Croal, B. L., Bachoo, P., Hillis, G. S., Cuthbertson, B. H., and Brittenden, J. (2008). N-terminal pro B-type natriuretic peptide is an independent predictor of postoperative myocardial injury in patients undergoing major vascular surgery. J. Vasc. Surg. 48, 912-917; discussion 917. doi: $10.1016 /$ j.jvs.2008.05.015

Rajagopalan, S., Croal, B. L., Reeve, J., Bachoo, P., and Brittenden, J. (2011). $\mathrm{N}$-terminal pro-B-type natriuretic peptide is an independent predictor of allcause mortality and MACE after major vascular surgery in medium-term follow-up. Eur. J. Vasc. Endovasc. Surg. 41, 657-662. doi: 10.1016/j.ejvs.2010. 12.017

Ridker, P. M., Cushman, M., Stampfer, M. J., Tracy, R. P., and Hennekens, C. H. (1997). Inflammation, aspirin, and the risk of cardiovascular disease in apparently healthy men. N. Engl. J. Med. 336, 973-979. doi: 10.1056/NEJM199704033361401

Roberts, S. E., and Goldacre, M. J. (2003). Time trends and demography of mortality after fractured neck of femur in an 
English population, 1968-98: database study. BMJ 327, 771-775. doi: 10.1136/bmj.327.7418.771327/7418/771.

Scrutinio, D., Passantino, A., Di Serio, F., Angiletta, D., Santoro, D., and Regina, G. (2011). High-sensitivity C-reactive protein predicts cardiovascular events and myocardial damage after vascular surgery. J. Vasc. Surg. 54, 474-479. doi: 10.1016/j.jvs.2011.01.041

Shroff, G. R., Akkina, S. K., Miedema, M. D., Madlon-Kay, R., Herzog, C. A., and Kasiske, B. L. (2012). Troponin I levels and postoperative myocardial infarction following renal transplantation. Am. J. Nephrol. 35, 175-180. doi: $10.1159 / 000335679$

Stang, A. (2010). Critical evaluation of the Newcastle-Ottawa scale for the assessment of the quality of nonrandomized studies in meta-analyses. Eur. J. Epidemiol/ 25, 603-605. doi: 10.1007/s10654-010-9491-z.

Stone, P. A., Schlarb, H., Campbell, J. E., Williams, D., Thompson, S. N., John, M., et al. (2014). C-reactive protein and brain natriuretic peptide as predictors of adverse events after lower extremity endovascular revascularization. J. Vasc. Surg. 60, 652-660. doi: 10.1016/j.jvs.2014.03.254

Stone, P. A., Thompson, S. N., Khan, M., Northfield, E., Schillinger, R., and Skaff, P. (2017). The Impact of biochemical markers on major adverse cardiovascular events and contralateral carotid artery stenosis progression following carotid interventions. Ann. Vasc. Surg. 38, 144-150. doi: 10.1016/j.avsg.2016. 08.004

Thygesen, K., Alpert, J. S., Jaffe, A. S., Chaitman, B. R., Bax, J. J., Morrow, D. A., et al. (2018). Fourth universal definition of myocardial infarction (2018). J. Am. Coll. Cardiol. 72, 2231-2264. doi: 10.1016/j.jacc.2018.08.1038
Tobias, A. (1999). Assessing the influence of a single study in meta-analysis. Stata Tech. Bull. 8:47.

Vetrugno, L., Costa, M. G., Pompei, L., Chiarandini, P., Drigo, D., Bassi, F., et al. (2012). Prognostic power of pre- and postoperative B-type natriuretic peptide levels in patients undergoing abdominal aortic surgery. J. Cardiothorac. Vasc. Anesth. 26, 637-642. doi: 10.1053/j.jvca.2012.01.018

Weber, M., and Hamm, C. (2006). Role of B-type natriuretic peptide (BNP) and NT-proBNP in clinical routine. Heart 92, 843-849. doi: $10.1136 /$ hrt.2005.071233

Winkel, T. A., Schouten, O., Hoeks, S. E., Voute, M. T., Chonchol, M., Goei, D., et al. (2010). Prognosis of vascular surgery patients using a quantitative assessment of troponin T release: is the crystal ball still clear? Eur. J. Vasc. Endovasc. Surg. 40, 739-746. doi: 10.1016/j.ejvs.2010.08.012.

Conflict of Interest Statement: The authors declare that the research was conducted in the absence of any commercial or financial relationships that could be construed as a potential conflict of interest.

Copyright (c) 2019 Zhang, Li, Li, Zeng and Liu. This is an open-access article distributed under the terms of the Creative Commons Attribution License (CC BY). The use, distribution or reproduction in other forums is permitted, provided the original author(s) and the copyright owner(s) are credited and that the original publication in this journal is cited, in accordance with accepted academic practice. No use, distribution or reproduction is permitted which does not comply with these terms. 